\title{
Effects of applied stress ratio on the fatigue behavior of additively manufactured porous biomaterials under compressive loading
}

\author{
Joep de Krijger ${ }^{\mathrm{a}}$, Calvin Rans ${ }^{\mathrm{b}}$, Brecht Van Hooreweder ${ }^{\mathrm{c}}$, Karel Lietaert ${ }^{\mathrm{d}, \mathrm{e}}$, Behdad Pouran ${ }^{\mathrm{f}, \mathrm{g}}$, \\ Amir A. Zadpoor ${ }^{\mathrm{f}, *}$ \\ a Department of Materials Science and Engineering, Faculty of Mechanical, Maritime, and Materials Engineering, Mekelweg 2, Delft 2628CD, The \\ Netherlands \\ baculty of Aerospace Engineering, Kluyverweg 1, Delft 2629HS, The Netherlands \\ ${ }^{c}$ Department of Mechanical Engineering, KU Leuven, Leuven, Belgium \\ d $3 D$ Systems - LayerWise NV, Leuven, Belgium \\ e Department of Materials Engineering, KU Leuven, Leuven, Belgium \\ ${ }^{\mathrm{f}}$ Department of Biomechanical Engineering, Faculty of Mechanical, Maritime, and Materials Engineering, Mekelweg 2, Delft 2628CD, The Netherlands \\ g Department of Orthopedics, UMC Utrecht, Heidelberglaan100, 3584CX Utrecht, The Netherlands
}

\section{A R T I C L E I N F O}

\section{Keywords:}

Cellular structures

bone grafting

orthopaedic implants

fatigue life

stress ratio

\begin{abstract}
A B S T R A C T
Additively manufactured (AM) porous metallic biomaterials are considered promising candidates for bone substitution. In particular, AM porous titanium can be designed to exhibit mechanical properties similar to bone. There is some experimental data available in the literature regarding the fatigue behavior of AM porous titanium, but the effect of stress ratio on the fatigue behavior of those materials has not been studied before. In this paper, we study the effect of applied stress ratio on the compression-compression fatigue behavior of selective laser melted porous titanium (Ti-6Al-4V) based on the diamond unit cell. The porous titanium biomaterial is treated as a meta-material in the context of this work, meaning that R-ratios are calculated based on the applied stresses acting on a homogenized volume. After morphological characterization using micro computed tomography and quasi-static mechanical testing, the porous structures were tested under cyclic loading using five different stress ratios, i.e. $R=0.1,0.3,0.5,0.7$ and 0.8 , to determine their $\mathrm{S}-\mathrm{N}$ curves. Feature tracking algorithms were used for full-field deformation measurements during the fatigue tests. It was observed that the S-N curves of the porous structures shift upwards as the stress ratio increases. The stress amplitude was the most important factor determining the fatigue life. Constant fatigue life diagrams were constructed and compared with similar diagrams for bulk Ti-6Al-4V. Contrary to the bulk material, there was limited dependency of the constant life diagrams to mean stress. The notches present in the AM biomaterials were the sites of crack initiation. This observation and other evidence suggest that the notches created by the AM process cause the insensitivity of the fatigue life diagrams to mean stress. Feature tracking algorithms visualized the deformation during fatigue tests and demonstrated the root cause of inclined $\left(45^{\circ}\right)$ planes of specimen failure. In conclusion, the R-ratio behavior of AM porous biomaterials is both quantitatively and qualitatively different from that of bulk materials.
\end{abstract}

\section{Introduction}

Additive manufacturing (AM) techniques such as selective laser melting (SLM) and electron beam melting (EBM) are increasingly used for manufacturing of bone substituting biomaterials and orthopaedic implants because of the many advantages they offer including design freedom, high precision, and the ability to produce parts directly from a CAD design without the need for molds (Thijs et al., 2010). Additively manufactured porous biomaterials based on lattice structures take full advantage of the possibilities offered by AM to maximize the bone regeneration performance of bone substituting biomaterials and the longevity of orthopaedic implants. The type of repeating unit cell and its dimensions can be chosen so as to adjust the mechanical properties of the resulting porous biomaterial (Heinl et al., 2008; Mullen et al., 2009) in order to more closely match the mechanical properties of bone. Achieving a closer match in the mechanical properties of bone and the implant is critical in mitigating risks associated with stress shielding (Ratner et al., 2013). In addition, the porosity of such

\footnotetext{
* Corresponding author.

E-mail address: a.a.zadpoor@tudelft.nl (A.A. Zadpoor).
} 
biomaterials can offer a large interconnected volume of space for bone ingrowth. The same interconnected volume of space could be used for drug delivery purposes to release growth factors (van der Stok et al., 2015) and/or anti-microbial agents (Amin Yavari et al., 2016). Furthermore, porous biomaterials often possess much larger surface area as compared to the equivalent solid shapes. The area of this surface can be either treated or coated so as to improve bone regeneration performance (Amin Yavari et al., 2014; Amin Yavari et al., 2014) or induce antibacterial properties.

During recent years, a lot of attention has been paid to manufacturing of porous structures based on designs that were impossible to produce using conventional methods. These structures are often made using EBM (Cansizoglu et al., 2008; Cheng et al., 2012; Hrabe et al., 2011; Li et al., 2012) or with SLM (Brenne et al., 2013; Gorny et al., 2011; Yan et al., 2012). In this study, we are particularly interested in SLM porous structures. SLM is a process that uses a laser to locally melt a thin layer of metal powder, which then solidifies in the desired cross-sectional shape. A new powder layer is placed on top of the partly solidified cross section, and the process is repeated until the full part is built. The most common material that is currently used for this manufacturing process is Ti-6Al-4V. Several researchers have manufactured porous metallic biomaterials aimed for orthopaedic applications using this titanium alloy (Chahine et al., 2008; Lin et al., 2007; Sallica-Leva et al., 2013).

As a consequence of the high temperature gradients experienced during the SLM process, unfavorable microstructures and residual stresses may develop in the material, resulting in fracture toughness and fatigue properties that are inferior to those observed for the parts made with conventional techniques (Cain et al., 2014; Leuders et al., 2013; Van Hooreweder et al., 2012). The unfavorable fatigue properties of SLM parts can, however, be improved by subsequent heat and surface treatment. Limited information (Hrabe et al., 2011; Amin Yavari et al., 2015; Amin Yavari et al., 2013; Zargarian et al., 2016) is available in the literature regarding the fatigue behavior of porous biomaterials made by additive manufacturing techniques in general and SLM in particular. In a recent work, it is shown how the porosity and the type of unit cell influence the compression-compression fatigue behavior of additively manufactured porous biomaterials (Amin Yavari et al., 2015; Amin Yavari et al., 2013). They found that the S-N curves normalized with respect to the yield stress of the porous structures conformed very well to one single power law for each type of unit cell. This shows that meta-materials are structures when their small-scale properties are considered, but they behave as materials when their homogenized macroscopic properties are studied (Van Hooreweder et al., 2012). Compression-compression loading is often (Amin Yavari et al., 2014; Hrabe et al., 2011; Li et al., 2012; Amin Yavari et al., 2015; Amin Yavari et al., 2013; Zargarian et al., 2016) used when studying the fatigue behavior of such additively manufactured porous biomaterials, because it is considered the most relevant mode of loading for bone-mimicking biomaterials.

Although there are advantages to considering the structural behavior of porous metal biomaterials in terms of relating performance to the mechanical behavior of the constituting material, treatment of the structure as a meta-material offers simplicity from a design and application standpoint. In line with this, the authors have decided for simplicity to adopt the meta-material viewpoint of porous metal biomaterials for this paper. This means that the R-ratios determined in this study are calculated based on applied stresses acting on the surface of homogenized material volume rather than the local physical stresses within the structure.

In mechanical fatigue, a standard sinusoidal stress cycle can be described by its minimum, maximum and mean stress, where the ratio between min and max is defined as the stress ratio, $R$. An $R$-value of 0.1 means that the maximum stress is ten times higher than the minimum stress. Previous studies on the fatigue behavior of AM porous biomaterials have all used one single $R$-value, i.e. $R=0.1$. However, porous biomaterials used in load-bearing orthopaedic applications are often subjected to various types of daily activities such as walking, hopping, and running. These activities are associated with different types of loading profiles (Caron et al., 2015; Hobara et al., 2014; Lohman et al., 2011) that might be different in their magnitude, frequency bands, and other characteristics. Designing load-bearing AM porous biomaterials therefore requires information regarding the fatigue response of those materials to different loading regimes including loading profiles with different stress ratios. Studies into the effects of R-ratio on the fatigue behavior of bulk Ti-6Al-4V have found that an increase in stress ratio for the same stress amplitude (i.e. increasing mean stress) results in a lower fatigue (Henry et al., 1994; Lindemann and Wagner, 1997; Nicholas, 2006). However, the dependency of the fatigue behavior on the stress ratio is known to be material-dependent and might be different for SLM porous biomaterials.

The current study aims to provide data and insight into the fatigue behavior of SLM porous titanium (Ti-6Al-4V) biomaterials when subjected to loading profiles with different applied stress ratios (from now on referred to as simply R-ratio). After morphological characterization using micro computed tomography (micro-CT), dry weighing, and Archimedes measurements, SLM porous structures based on the diamond unit cell were mechanically tested under compression to determine their quasi-static mechanical properties. The compressioncompression S-N curves of the same porous structures were then determined experimentally using loading profiles with different Rratios.

\section{Materials and methods}

\subsection{Additive manufacturing}

Test samples were manufactured using SLM (3D Systems) from Ti6Al-4V-ELI powder according to ASTM F3001. This alloy has a theoretical density of $4.42 \mathrm{gcm}^{-3}$. The build chamber had an inert Ar atmosphere with an oxygen level below $50 \mathrm{ppm}$. The samples were built using a similar procedure and similar parameters as described in our previous studies (Amin Yavari et al., 2016; Amin Yavari et al., 2014; Amin Yavari et al., 2014; Amin Yavari et al., 2015; Amin Yavari et al., 2013; Ahmadi et al., 2015). The samples were built on top of a solid titanium build plate from which they were subsequently removed using wire electrical discharge machining (EDM).

Repeating the diamond unit cell in all directions created the porous structures of the cylindrical test specimens with diameter of $15 \mathrm{~mm}$ and length of $20 \mathrm{~mm}$. The front view of a test sample and the basic unit cell are displayed in Fig. 1. The nominal (i.e. designed) porosity of the specimens was $80 \%$. STL files were created using the Magics software from Materialise (Leuven, Belgium). 3D Systems' DMP Explorer software was used for slicing and hatching of the stl file.

\subsection{Morphological characterization}

The morphological features of the AM porous structures were characterized using dry weighing, Archimedes measurements, and micro-CT scans. For the Archimedes measurements, the test procedure

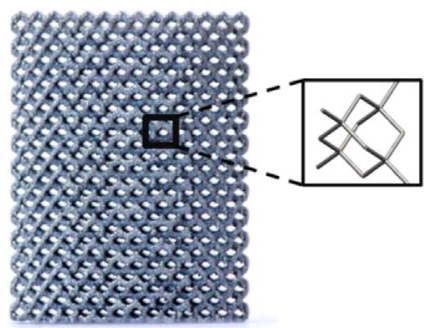

Fig. 1. Diamond unit cell porous structure, Dimensions: $15 \times 20 \mathrm{~mm}$. 
described in ASTM B311 (B311, 2013) was followed. This standard describes the procedure for measuring the density of powder metallurgy materials. A batch with a minimum of five samples was first weighed on a balance with a precision of $0.0001 \mathrm{~g}$ (Denver Instruments AA-160). The 'dry porosity' was then calculated by dividing the actual weight by the theoretical weight of the macro volume. The sample was then submerged in pure ethanol and weighed again, from which the actual volume could be calculated. The Archimedes porosity was then calculated by dividing this actual volume by the total macro volume of the sample.

Micro-CT scans were performed using a Caliper LifeSciences Quantum FX $\mu$-CT scanner. Five samples were scanned for 120 seconds at $90 \mathrm{kV}$ and $180 \mu \mathrm{A}$, at a resolution of $48 \mu^{3}$ per voxel. The image processing method is similar to previous studies from (Amin Yavari et al., 2015), while a more detailed description of the image processing procedures is provided in (Ahmadi et al., 2015). The images were processed using the software package Image-J. This was done by first applying an automatic local threshold (Niblack, radius 15) to create a binary image. The same thresholding method was applied for all microCT slices. From this binary image, the overall porosity and average pore and strut sizes were determined using the volume fraction algorithm available in the BoneJ plugin.

\subsection{Quasi-static mechanical testing}

The test procedure for the static compression test was based on ISO 13314:2011 (ISO, ISO 13314, 2011). A Zwick Z100 (100kN) test system with compression plates was used for these tests. A constant deformation rate of $1.2 \mathrm{~mm} / \mathrm{min}$ was applied until $60 \%$ strain after which the test was automatically stopped. A total of three specimens were tested. The following parameters were calculated using the obtained stress-strain curves: maximum stress $\left(\sigma_{\max }\right)$ and its corresponding strain $\left(\mathrm{e}_{\mathrm{max}}\right)$, the plateau stress $\left(\sigma_{\mathrm{pl}}\right)$ which is calculated as the arithmetical mean of the stresses between $20 \%$ and $40 \%$ strain, the quasi-elastic gradient $\left(\mathrm{E}_{20-70}\right)$ which is the elastic straight line between $20 \%$ and $70 \%$ of the plateau stress, the compressive offset stress $\left(\sigma_{\text {off }}\right)$ which is the compressive stress at $0.2 \%$ plastic strain obtained from the quasi-elastic gradient and the energy absorption (E.A.) which is the area under the stress-strain curve up to $50 \%$ strain. The offset stress is considered to represent the yield stress of the porous structures to enable comparison with other studies. As mentioned in the introduction, all stresses mentioned in this paper are based on a meta-material viewpoint of the porous biomaterial, and hence based on the total circular area of the cylindrical sample with a diameter of $15 \mathrm{~mm}$.

\subsection{Fatigue testing}

A fatigue test protocol similar to the ones used in our previous studies was used (Amin Yavari et al., 2015; Amin Yavari et al., 2013). $\mathrm{S}-\mathrm{N}$ curves were constructed by measuring the force-controlled fatigue life of porous structures at ten different maximum stresses between $20 \%$ and $90 \%$ of the yield (offset) stress. Each test was repeated at least two times. If the difference between cycles to failure was larger than $40 \%$ of their average value, a third sample was tested. The fatigue tests were carried out at a stress ratio of $R=0.1,0.3,0.5$ and 0.7 , resulting in an SN curve for each stress ratio. An exponential fit to the $\mathrm{SN}$ curves was carried out using the MATLAB curve-fitting tool using a nonlinear least squares criterion. The $\mathrm{S}-\mathrm{N}$ curves were then combined to create a constant life (fatigue) diagram, which can be used to visualize the mean stress behavior of the samples and to compare this with the literature. A few extra tests were conducted at a stress ratio of $R=0.8$ to obtain more data points for constructing the constant life diagram. All tests were carried out on an MTS $100 \mathrm{kN}$ hydraulic test machine, at a loading frequency of $15 \mathrm{~Hz}$ with a sinusoidal wave shape. The tests were continued until failure of the specimen, however exceeding $10^{6}$ cycles was regarded as a run-out. The run-out tests are marked with an arrow

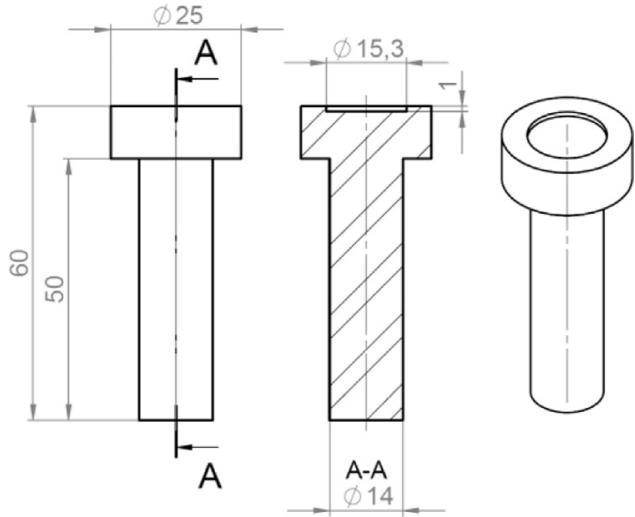

Fig. 2. Specimen fixture for fatigue tests, dimensions are in $\mathrm{mm}$.

in the final S-N curve. The point of failure was defined by an increase in displacement of $2 \mathrm{~mm}$. To prevent the sample from moving and making sure that the sample was aligned properly during the tests, a sample holder as displayed in Fig. 2 was used. The specimens were placed between two sample holders.

\subsection{Full-field deformation measurements}

For a selected number of fatigue tests, full-field deformation measurements were performed using a digital camera system (Optomotive Velociraptor) at an interval of 100 cycles. In order to obtain images at the same point in every cycle, the testing machine was programmed to stop at the maximum load after every 100th cycle, and send a signal to the camera system that then triggered the camera shutter after which the cycle continued. This process was repeated until the sample failed. The obtained images were then processed with MATLAB to visualize the local displacement values of the sample. This was done by using a feature detection algorithm (Speeded Up Robust Features, detectSURFFeatures) from the Computer Vision System Toolbox that looks for matching features in two images. A close-up of the sample from the image correlation process is displayed in Fig. 3. In this picture, the two compared images are placed on top of each other with the first picture in red, and the displaced image in blue. Corresponding features are then marked with a yellow line to indicate the displacement. The distance in pixels of the two successive pictures is then calculated after which an outlier filter is applied to remove unwanted points. Some of the corresponding features that the MATLAB algorithm recognizes are extremely far away. For example, a point at the upper left corner of the sample may be corresponded to a

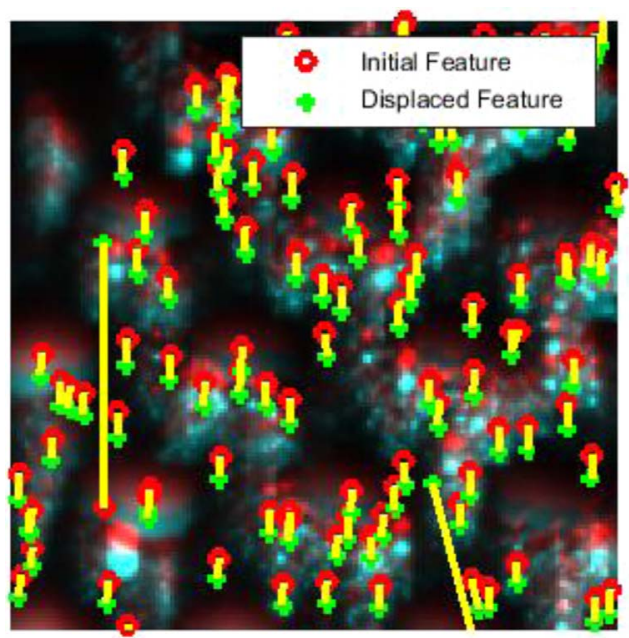

Fig. 3. Detection of corresponding points in the feature tracking code. 
point at the bottom. Such correspondences in the feature tracking algorithm would result in impossibly and unrealistically large displacements. An outlier filter was therefore applied to removes such 'impossible' correspondences. The distances were then normalized by dividing them by the initial length of the sample that was measured with a caliper before each test. The displacement field was then visualized by applying a colored marker to the displaced point, ranging from blue to red with an increasing magnitude of the deformation.

\subsection{Constant fatigue life diagrams}

To study the effect of stress amplitude and mean stress on fatigue life, it is customary to plot the so-called constant life diagrams. In a constant life diagram, the combination of stress amplitude and mean stress is displayed for a specific number of cycles that lead to failure.

A common way of comparing or fitting data in constant life diagrams is by plotting the modified Goodman relationship:

$\sigma_{a}=\sigma_{-1}\left(1-\frac{\sigma_{m}}{\sigma_{u}}\right)$

where $\sigma_{-1}$ is the fatigue life for complete reverse loading (i.e. $R=-1$ ), $\sigma_{m}$ is the mean stress, and $\sigma_{u}$ is the ultimate tensile strength of the material. This relationship predicts a linear descent of the constant life from the fully reversed loading $\left(R=-1, \sigma_{m}=0\right)$ to the static ultimate stress of the material, where the amplitude is zero and the mean stress is equal to the ultimate stress.

The choice of fully reversed loading $(R=-1)$ as a datum point in the Goodman relationship stems from the prevalence of rotating beam fatigue testing as a means to measure the fatigue resistance of materials at the time. This test was relatively simple and efficient at applying large numbers of fatigue cycles in a short period of time; however, it was limited by the fact that it could only test fully reversed loading. It is possible to reformulate this relationship in terms of an alternative datum while keeping the linear nature of the Goodman relation. Doing so, Eq. 1 can be reformulated as:

$\sigma_{a}=\frac{\sigma_{a R} \sigma_{u}}{\sigma_{u}-\sigma_{m R}}\left(1-\frac{\sigma_{m}}{\sigma_{u}}\right)$

where the subscript $R$ in $\sigma_{a R}$ and $\sigma_{m R}$ indicates the R-ratio of the newly selected datum. For this study, as fully reversed loading conditions were not tested, a datum of $\mathrm{R}=0.1$ was selected. This choice was made as the conditions of mean stress and amplitude stress at this R-ratio were closest to the fully reversed loading tested in this study. When using this equation within this study, it will be referred to as the Modified Goodman relation to highlight the change in datum.

Another common comparison that is used in fatigue diagrams is the Gerber parabola, which has the same axis intersections as the modified Goodman relation but assumes a parabolic relation for the descent. In order to compare these graphs from the literature with the test results of this study, three curves from the ASM international Fatigue data book (Henry et al., 1994) were digitized using a plot digitizer tool (Rohatgi, 2015).

\subsection{Optical microscopy}

The fracture surfaces were examined using an optical microscope (Keyence VHX-5000 series Digital microscope, lens: Z250 dual-light high magnification zoom lens, 250-2500X).

\section{Results}

\subsection{Morphological characterization}

The actual porosities of the porous structures measured by various techniques were all close to the nominal (design) porosity, i.e. $80 \%$
Table 1

Morphological and compressive mechanical properties (mean \pm standard deviation) of the porous structures.

\begin{tabular}{ll}
\hline Property & Measured value \\
\hline Dimensions, D x L (mm) & $15 \times 20$ \\
Unit cell size (mm) & 1.5 \\
Dry weight (g) & $3.114 \pm 0.079$ \\
Porosity, dry weighing (\%) & $80.1 \pm 0.5$ \\
Porosity, Archimedes (\%) & $79.2 \pm 0.6$ \\
Porosity, Micro-CT (\%) & $79.8 \pm 0.3$ \\
Pore size, Micro-CT $(\mu \mathrm{m})$ & $765 \pm 112$ \\
Strut diameter, Micro-CT $(\mu \mathrm{m})$ & $306 \pm 60$ \\
$\sigma_{\max }(\mathrm{MPa})$ & $55.6 \pm 0.8$ \\
$\mathrm{e}_{\max }(\%)$ & $6.3 \pm 0.7$ \\
$\sigma_{\text {off }}\left[\sigma_{\mathrm{y}}\right](\mathrm{MPa})$ & $43.0 \pm 2.1$ \\
$\sigma_{\mathrm{pl}}(\mathrm{MPa})$ & $35.3 \pm 2.2$ \\
$\mathrm{E}_{20-70}(\mathrm{GPa})$ & $1.36 \pm 0.46$ \\
E.A. $\left(\mathrm{MJm}{ }^{-3}\right)$ & $17.3 \pm 0.6$
\end{tabular}

(Table 1). There was relatively small standard deviation in the morphological properties of the different samples such as porosity ( $<0.5 \%)$, strut diameter $(60 \mu \mathrm{m},<20 \%$ of the mean value), and pore size $(112 \mu \mathrm{m},<15 \%$ of the mean value) (Table 1$)$.

\subsection{Quasi-static mechanical properties}

There was little variation in the measured mechanical properties of the porous structures (Table 1). The struts of the specimens failed close to the compression plates, either at the top or bottom, after which they gradually crushed from this location (Fig. 4a). An inclined failure line (45 degrees) was observed during the gradual breakdown (Fig. 4a). Struts that were in contact with the compression plate failed first after which the sample gradually crushed from that position.

\subsection{Fatigue testing}

For the same values of normalized stress (max. applied stress / yield stress), loading under higher R-ratios resulted in greater number of cycles to failure (Fig. 5a). This is to be expected since for any given maximum stress, the stress amplitude lowers when the R-ratio increases. The S-N curves plotted on a double logarithmic scale show a similar slope for R-ratios 0.7 and 0.8 and for R-ratios 0.1 and 0.3 (Fig. 5b). For every R-ratio, the S-N data can be very well represented by exponential trendlines $\left(R^{2} \approx 0.98\right)$ (Table 2). Plotting the number of cycles against the stress amplitude resulted in a single trend for all tested specimens regardless of the stress ratio under which they were tested (Fig. 6). However, there was a slight decrease in the fatigue life for high stress ratios that could be more clearly seen for stress levels below $0.1 \sigma_{\mathrm{y}}$ (Fig. 6).

One way of presenting the gradual failure during the fatigue life of this type of samples is to plot the stiffness of the specimens in function of the percentage of the total fatigue life (Fig. 7). The stiffness was calculated by dividing the maximum force during a cycle, by the displacement measured by the fatigue machine (both data is measured directly by the machine). So the displacement describes the distance that a sample is compressed at the maximum force during a cycle. This displacement increases during the fatigue test, which can be described as a stiffness decrease. This quantity represents the relative stiffness and this value is different from the actual stiffness, because the displacement at the minimum force also increases over the fatigue life. It can nevertheless be used to observe the gradual failure of the samples. The stiffness values were normalized with respect to the maximum stiffness to enable comparison between different load cases. All stress ratios showed comparable stiffness degradation behaviors (Fig. 7). The final failure occurred at a lower stiffness decrease for maximum normalized stresses above 0.4 or mean normalized stresses 


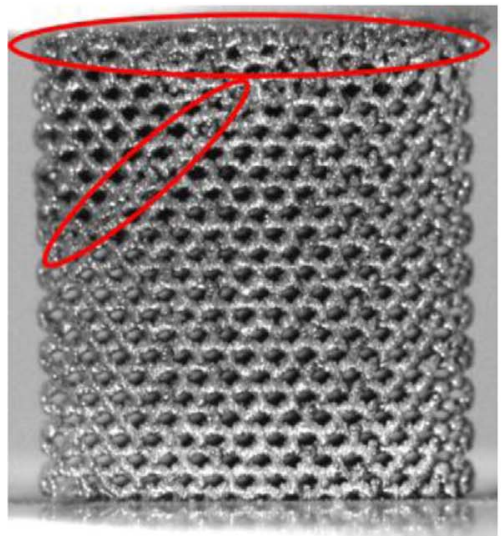

a)

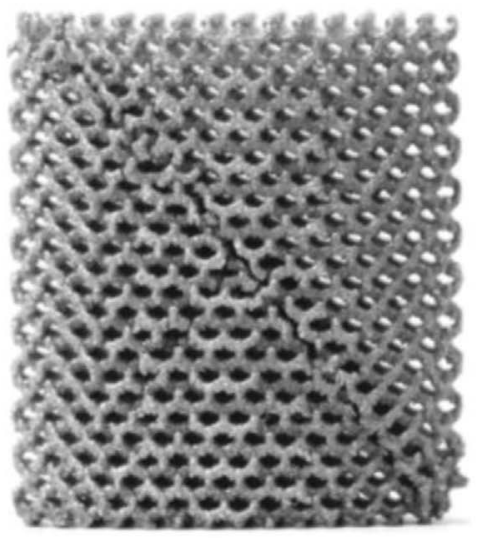

b)

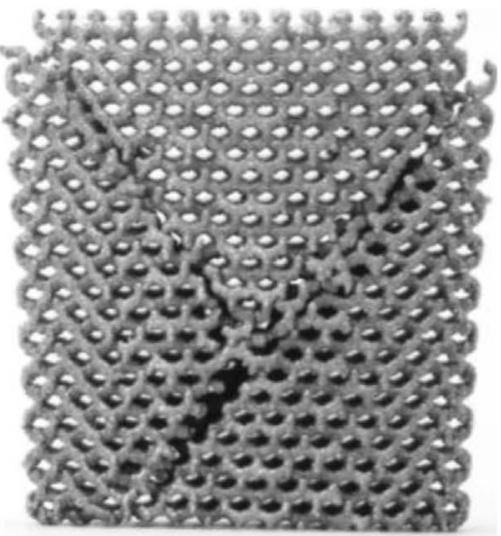

c)

Fig. 4. Typical sample failure at static compression (a), fatigue failure at one (b) or two directions (c).
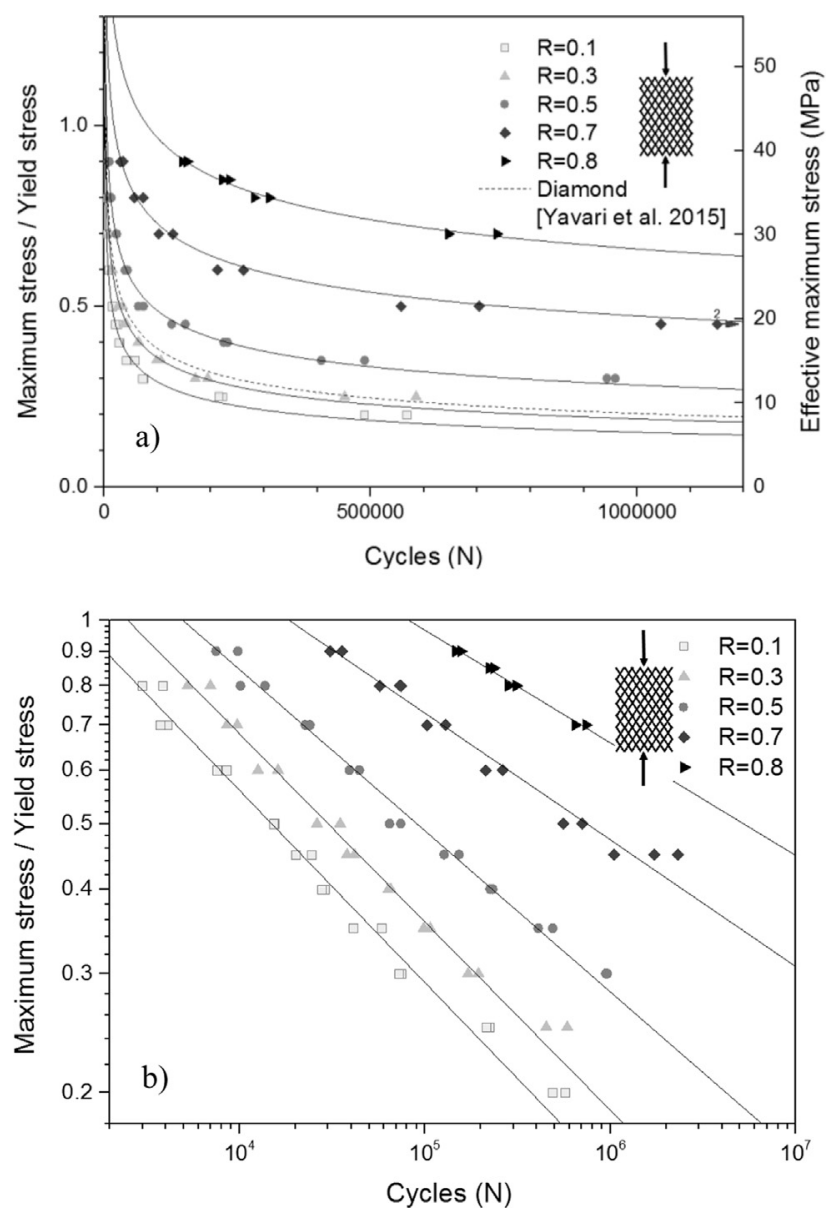

Fig. 5. Obtained SN-curves at different stress ratios on a linear (a) and logarithmic (b) scale. The number 2 in subfigure (a) indicates the number of run-out specimens.

Table 2

Exponential curve fitting parameters $\left(y=a x^{b}\right)$.

\begin{tabular}{llll}
\hline & $a$ & $b$ & $R^{2}$ \\
\hline$R=0.1$ & 7.703 & -0.2846 & 0.9854 \\
$R=0.3$ & 8.848 & -0.2787 & 0.9876 \\
$R=0.5$ & 7.620 & -0.2387 & 0.9880 \\
$R=0.7$ & 6.318 & -0.1876 & 0.9792 \\
$R=0.8$ & 6.597 & -0.1668 & 0.9886 \\
\hline
\end{tabular}

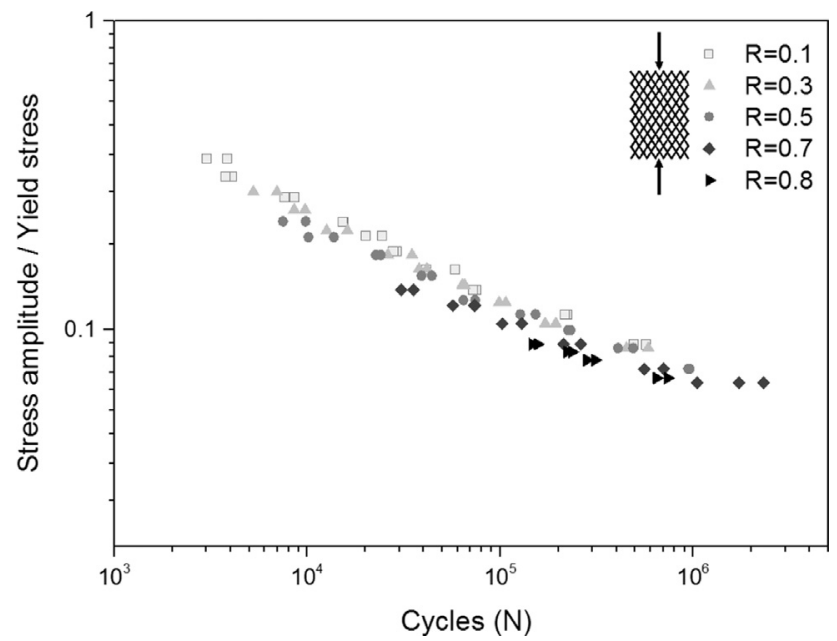

Fig. 6. Normalized stress amplitude vs. number of cycles to failure.

higher than 0.3 (Fig. 7). The number of cycles to failure of the experiments presented in Fig. $7 \mathrm{a}$ varied between $\approx 130.000$ and 225.000 for $R=0.3$ to $\mathrm{R}=0.8$, and was around 560.000 cycles for $R=0.1$. An overview of the normalized loads for each test is displayed in the table in the graph.

Further analysis showed that the specimen tested at the lowest amplitude $(R=0.7)$ had a constant rate of stiffness degradation comparable to the other tests, but over a longer period of the total fatigue life and a more rapid decrease near the end (Fig. 7b). The repeated tests that were performed under the same stress conditions showed the same behavior. The tests with stress amplitudes of $0.15 \sigma_{\mathrm{y}}$ and $0.245 \sigma_{\mathrm{y}}$ showed a very similar rate of stiffness degradation (Fig. 7b).

The test that is marked with $R=0.3 \mathrm{a}$ in Fig. $7 \mathrm{~b}$, shows a stiffness increase during the test, which dropped again after around $50 \%$ of the fatigue life. This behavior occurred for four other specimens with different loading conditions. The total number of cycles did not deviate much from the repeated tests at the same conditions.

\subsection{Feature tracking for deformation measurement}

The full-field deformation measurements performed using feature tracking algorithms clearly showed an area of high strain concentration (highlighted by an abrupt change in deformation contour) with angles similar to what was ultimately seen in the failure lines of the specimens (Figs. 4, 8-10). The white line marks the direction of the final failure of the samples (Figs. $8-10)$. At $50 \%$ of the fatigue life $(R=0.1)$, the 

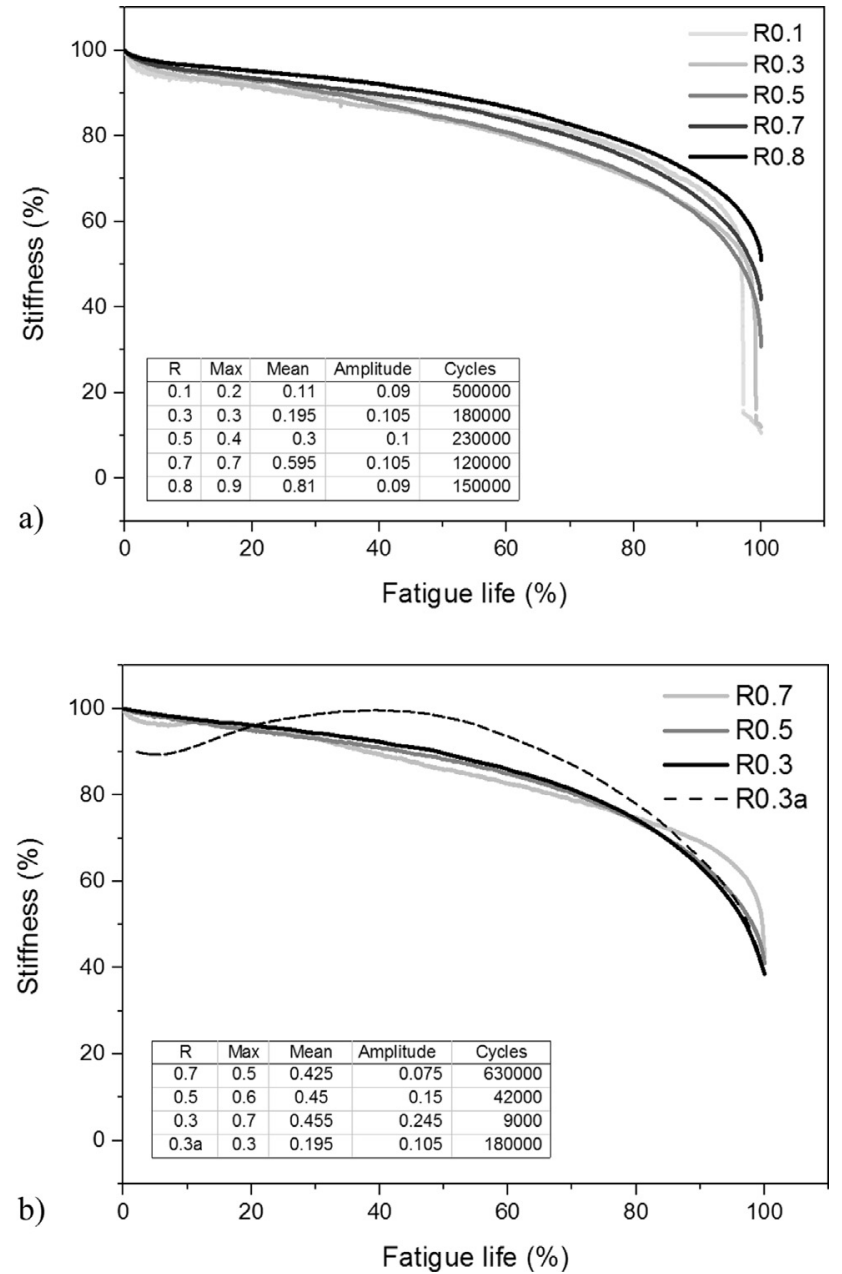

Fig. 7. Stiffness decay over lifetime for a constant stress amplitude (a) and increasing amplitudes (b).

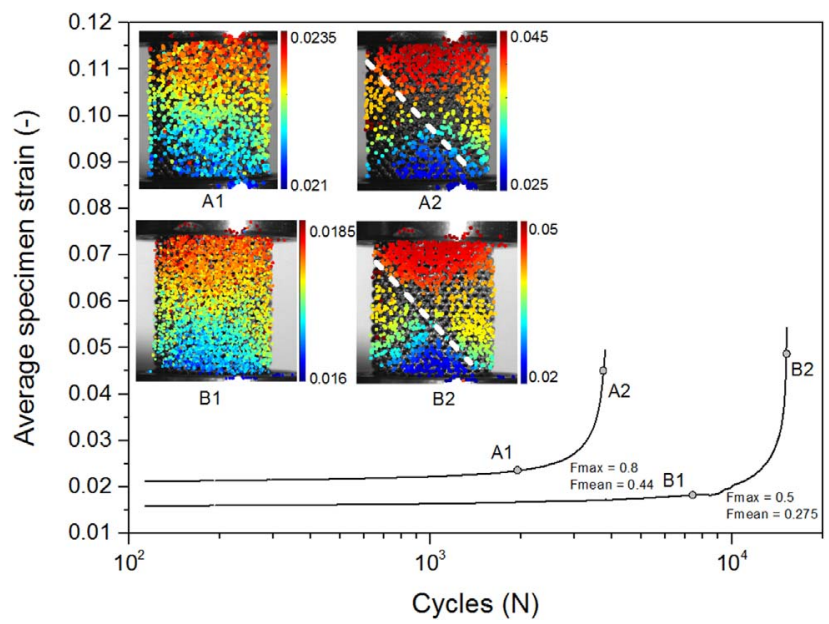

Fig. 8. Strain development during fatigue tests at $R=0.1$.

deformation distributions already indicate increased deformations at an angled orientation, which was more pronounced for the specimens subjected to fatigue loading with lower maximum stress (Fig. 8).

Three different stress ratios with approximately the same amplitudes were compared (Fig. 10). For specimens B and C, a cross shape could already be observed halfway the fatigue life (Fig. 10). All three specimens showed a similar pattern of stiffness degradation over the fatigue life, except for the test at $R=0.1$, which showed a higher rate of

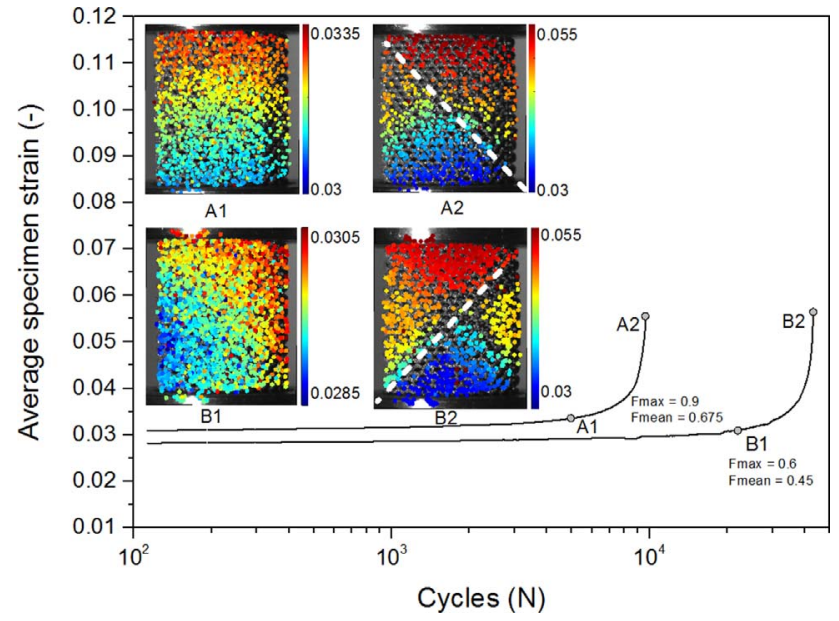

Fig. 9. Strain development during fatigue tests at $R=0.5$.

stiffness degradation over the fatigue life (Fig. 10). The displacement values measured using feature tracking algorithms were in good agreement with those measured using the fatigue test machine (Fig. 10).

Comparisons between the feature tracking results for different load cases showed only small differences. At high maximum stresses the deformation distribution was more horizontal, but this did not affect the final failure direction. Also no apparent differences were visible between the tests at the same stress ratios, except for one test that is displayed in Fig. 9, where a clear upper and lower triangular part was visible in the deformation distribution which did not correspond with the final failure direction. Looking only at inset figure B1, one might expect a failure direction that starts in the upper left corner, going to the lower right corner, because the strains in the upper right corner of the sample are slightly larger than the lower left The final failure occurred in the other direction as seen in inset figure B2, indicated with the with a dashed line. This 'change' occurred gradually during the cycles between B1 and B2.

\subsection{Constant fatigue life diagrams}

The constant life diagrams for the tests performed in the current study were plotted by fitting exponential fits to the test data (Fig. 11) and are compared with the typical behavior of bulk Ti-6Al-4V reported in the literature (Henry et al., 1994) (Fig. 12). When the normalized mean stress increases from 0.2 to 0.9 , the normalized stress amplitude slightly decreases for the constant life diagrams of 50.000 and 100.000 cycles (Fig. 11). Increased values of mean stress did not result in any notable decrease of the stress amplitude for the constant life diagram of 500.000 cycles (Fig. 11). The fit of the modified Goodman relation and the Gerber parabola were not very good for the obtained data set. The modified Goodman relation only fitted our test data at our selected datum of $\mathrm{R}=0.1$ (which by definition it must fit exactly) and its close proximity up to $\mathrm{R}=0.3$ (Fig. 11). The Gerber parabola fared better; however, a clear parabolic trend in the obtained data set was not visible (Fig. 11).

\subsection{Fatigue fracture analysis}

During all fatigue tests, failure occurred at a $45^{\circ}$ angle (Fig. $4 \mathrm{~b}-\mathrm{c}$ ). Powder from the SLM process was visible on the surface of the struts and the overall appearance was irregular (Fig. 13a). Observation of the fracture surface showed that multiple fatigue cracks were initiated from the rough surface (Fig. 13b). Most samples failed along one direction, while a few samples failed at both +45 and -45 degrees, resulting in a triangular or cross-shaped failure (Fig. 4b-c). No relation was found 

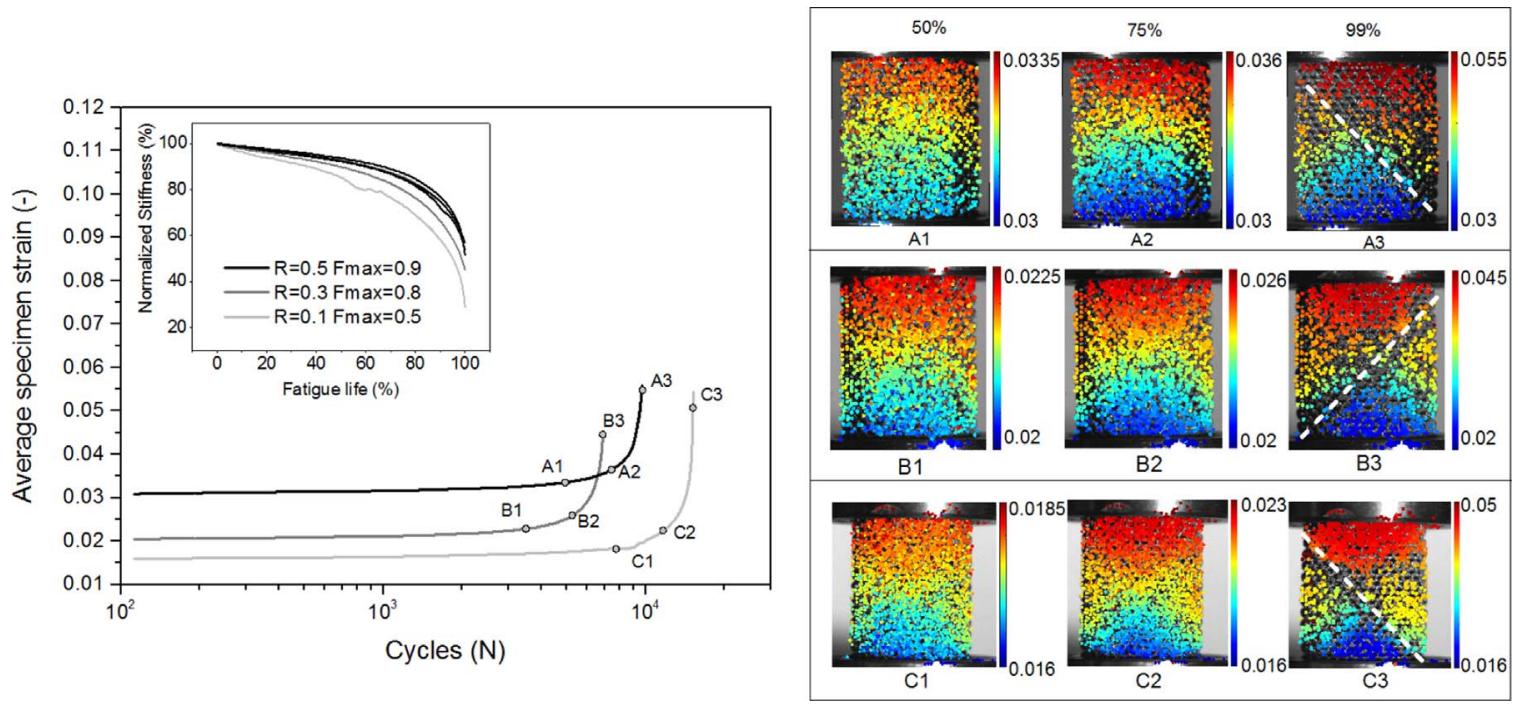

Fig. 10. Strain development during Fatigue tests at equal amplitudes and different mean stresses $\left(0.675 \sigma_{\mathrm{y}}(A), 0.52 \sigma_{\mathrm{y}}(\mathrm{B}), 0.275 \sigma_{\mathrm{y}}(\mathrm{C})\right)$.

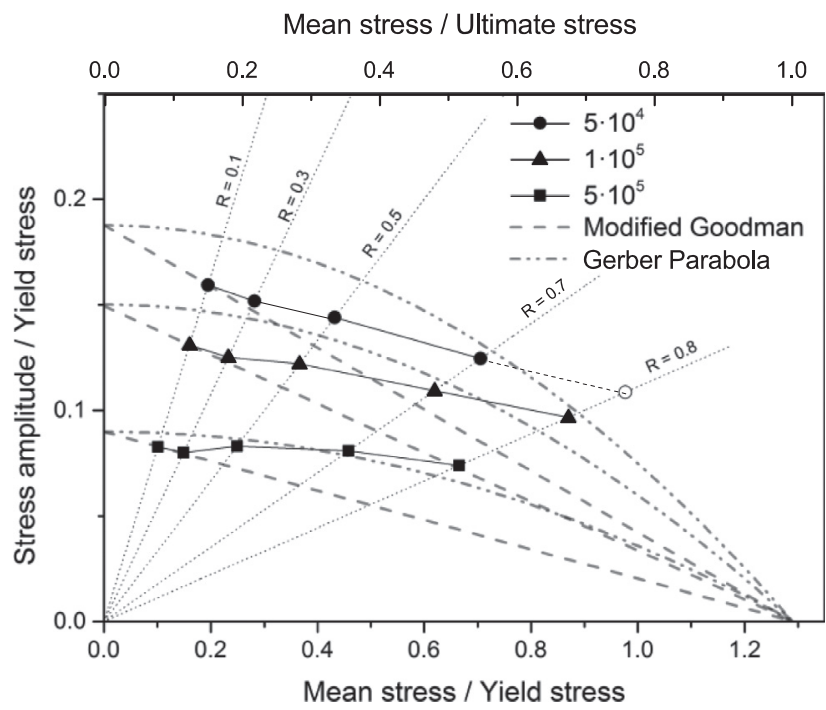

Fig. 11. Constant life diagram constructed form test results.

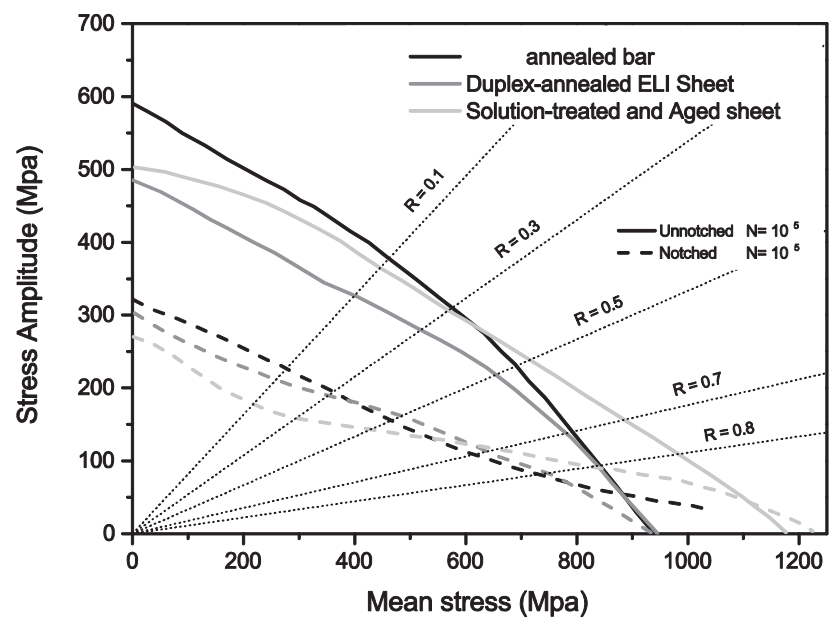

Fig. 12. Constant life diagram comparing different Ti-6Al-4V compositions from the data presented (from different sources) in reference (Henry et al., 1994).

between the applied loads and this failure appearance.

\section{Discussion}

Compression-compression fatigue tests do normally not cause fatigue failure in continua, but several studies (Hrabe et al., 2011; Amin Yavari et al., 2015; Amin Yavari et al., 2013) have shown that porous structures fail under compressive-compressive cyclic loading. The fatigue tests performed in the current study confirmed the previous findings on porous materials that the $\mathrm{S}-\mathrm{N}$ behavior of compressive cyclic loading on porous materials results in the same $\mathrm{S}-\mathrm{N}$ trend that is characteristic for tension-tension cyclic loading of the parent material. The reason for this is that the compressive loading and the compressive stresses described in this work refer to the homogenized volume of the meta-material (i.e. the cylindrical specimen) and not the struts. When looking at the fatigue samples as structures, it becomes clear that the struts or beams are actually loaded in a combination of cyclic bending and cyclic axial compressive stresses (Van Hooreweder et al., 2016). The cyclic loading of the structure in compression-compression gives rises to both compressive and tensile stresses in the struts. It is most likely that these tensile stress components cause the fatigue failure of the struts, ultimately leading to fracture of the porous sample. This is illustrated in Fig. 14 that shows an arbitrary strut of the diamond unit cell, its hyperstatic boundary conditions similar to the free bodydiagram as presented in (Ahmadi et al., 2014), and an approximation of the normal stress distributions that occur (Van Hooreweder et al., 2016).

The axial compressive stress $S_{A}$ and the bending stress $S_{B}$ can be calculated using strut diameter $d$, strut length $L$, strut area $A$ and moment of inertia $I$. For these diamond unit cells with properties shown in Table 1 , the angle $\theta=35,26^{\circ}$ represents the orientation of the strut force $F$, as explained more in detail in (Van Hooreweder et al., 2016; Ahmadi et al., 2014).

$S_{B}=\frac{F L d \cos \theta}{4 I} \cong 94.3 F$ with $I=\frac{\pi d^{4}}{64}$

$S_{A}=\frac{F \sin \theta}{A} \cong 7.8 F$ with $A=\frac{\pi d^{2}}{4}$

$S_{1}=F\left(\frac{L d \cos \theta}{4 I}-\frac{\sin \theta}{A}\right) \cong F(94.3-7.8)$

$S_{2}=F\left(\frac{L d \cos \theta}{4 I}+\frac{\sin \theta}{A}\right) \cong F(94.3+7.8)$

It is important to notice that these formulations are based on several assumptions including linear material behavior, constant and 

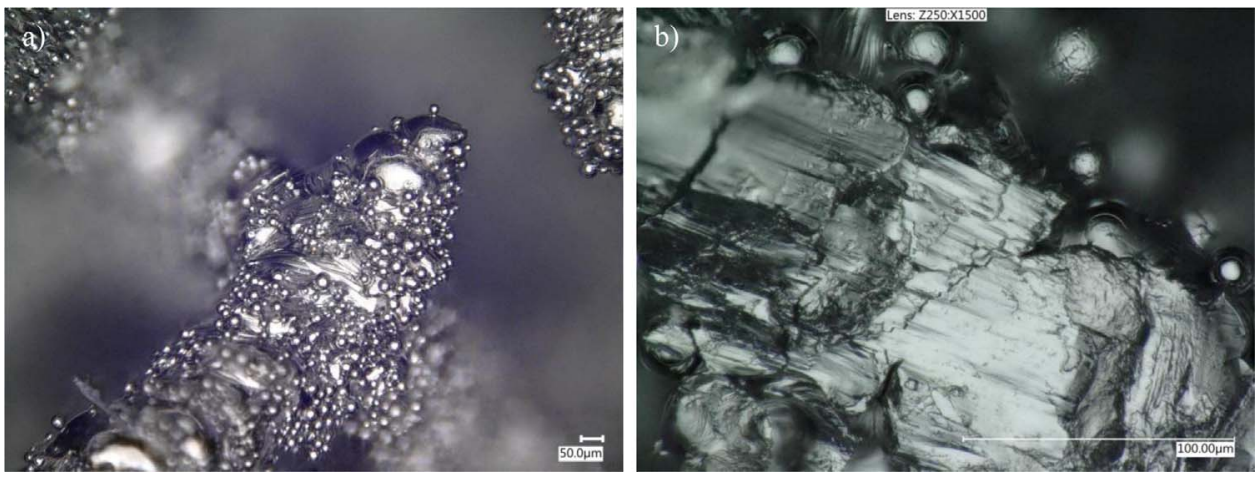

Fig. 13. Close-up of strut at 250x magnification (a) Fracture Surface with crack initiated from notch at a magnification of $1500 x$.

uniform geometrical properties $(d, L)$, absence of stress concentrations and simplified boundary conditions, and are therefore useful only for providing insight into the general state of stress in the struts and not accurate estimation of stress values. It is for instance shown that the load angle $\theta$ has an important influence on the ratio of uniform compressive stress $S_{A}$ compared to the bending stress $S_{B}$. For this particular sample, and for assuming $\theta$ constant, it is clear from Eqs. 3 and 4 that the bending stress $S_{B}$ is much larger than the axial compressive stress $S_{A}$, which leads to significant tensile stresses $S_{1}$ on the surface of the struts. Although the total tensile stress $S_{1}$ is smaller in absolute value than the total compressive stress $S_{2}$, it is most likely that $S_{1}$ eventually causes the strut to fail in fatigue. This is also confirmed by analyzing the fractured surfaces, were cracks were mainly found in the upper parts of the struts (Fig. 13).

As indicated earlier, the R-ratio in this work is calculated from the minimum and maximum loading that is applied on the whole porous structure and not from the actual local minimum and maximum stresses occurring in a critically loaded point of a strut. When assuming constant load angle $\theta$, the relation between load $F$ and stress $S$ is constant, as shown in Eqs. 5 and 6, and hence the ratio of loading is equal to the ratio of local stress. This is not the case when load angle $\theta$ is not constant. When the sample is subjected to cyclic loading with high load and hence high mean load, the sample will deform, the average load angle $\theta$ will decrease and hence $S_{B}$ will gain more importance over $S_{A}$. This geometric non-linearity clearly influences the state of stress, and hence also the stress ratio. This effect might be significant depending on parent material, unit cell type and sample porosity. In the current study, this effect was not taken into account, and R-ratios are hence properties of the structure, and not of the material.

S-N curves shifted upwards when loading was performed under higher stress ratios (Fig. 5a). This is a result of the lower stress amplitudes experienced for higher stress ratios given the fact that the tests were based on predefined maximum loads. It was also observed that the slope of the S-N curves plotted on a logarithmic scale decreases for higher stress ratios (Fig. 5b), suggesting that a relatively small change in the maximum stress influences the fatigue life more strongly for higher stress ratios. Stress amplitude is clearly the dominant factor in determining the $\mathrm{S}-\mathrm{N}$ curve (Fig. 6).

Fatigue diagrams of bulk Ti-6Al-4V from literature all show similar behavior, with a clear decline of the stress amplitude when the mean stress is increased (Fig. 12). The lines in Fig. 12 represent $10^{5}$ cycles of both notched and smooth specimens for three different heat-treatments of bulk Ti-6Al-4V. A significant $R$-ratio effect is observed for this material, which shows a similar behavior as predicted by the Gerber or modified Goodman relation. The R-ratio behavior of the AM porous biomaterials studied here (Fig. 11) follows neither the Goodman or Gerber predictions and is therefore not only quantitatively but also qualitatively different from that of the bulk Ti-6Al-4V material (Fig. 12). These observations mark clear differences between the fatigue behavior of $\mathrm{AM}$ porous biomaterials and that of conventional bulk materials.

The relatively low sensitivity of the constant life diagrams obtained in the current study to mean stress can be explained by increased surface roughness and the notches that are present in the struts of AM porous biomaterials. In bulk Ti-6Al-4V materials, notches not only reduce fatigue strength but also reduce the sensitivity of the fatigue life to mean stress (Fig. 12). Lanning (Lanning et al., 2005) found similar behavior for notched specimens as compared to smooth specimens. A method to quantify the notch effect is discussed in (Nicholas, 2006), where the (un-notched) data of the amplitude in a constant life diagram is reduced by a stress concentration factor, $k_{t}$, or a fatigue notch factor, $k_{f}$. Large values of these factors significantly reduce the slope of the curve, thereby reducing the sensitivity of the fatigue life to the mean stress, which results in constant life diagrams that are similar to the notched behavior of bulk Ti-6Al-4V (Fig. 12) and the constant life diagrams obtained in the current study for 50.000 and 100.000 cycles (Fig. 11). The statement that notches could be the cause of the low mean stress sensitivity of the AM porous biomaterials considered here is supported by the fact that fatigue cracks seemed to initiate from the notches present in the struts of the porous structure (Fig. 13a). The imperfections caused by the manufacturing process have been shown

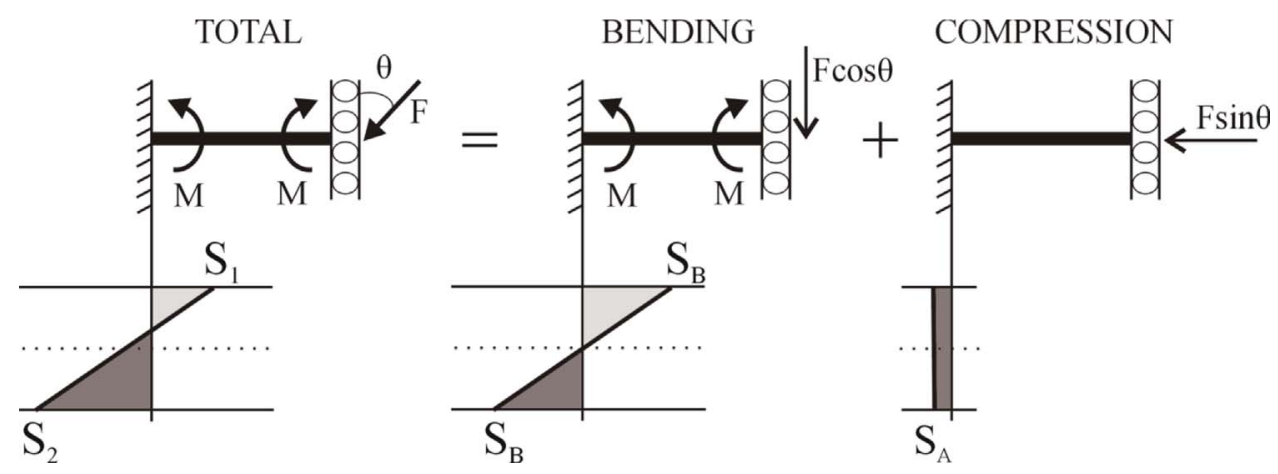

Fig. 14. Approximation of loading and normal stress distribution of one single strut of the diamond unit cell. 
to significantly influence the mechanical properties of various types of materials including additively manufactured (Campoli et al., 2013) and machined (Zadpoor et al., 2008) materials. The surface roughness of the struts is dependent on the AM process including both laser processing parameters and the size and shape of the powder that is used in the powder bed. Increased surface roughness of the struts could cause stress concentrations and act as crack initiation sites (Amin Yavari et al., 2014). One approach for improving the fatigue resistance of AM porous biomaterials could therefore be to optimize the manufacturing process including the laser processing parameters and the characteristics of the powder including its size distribution and flowability such that the size and distribution of the surface roughness are more effectively controlled.

The displacement fields obtained by feature tracking show that it is possible to visualize the failure direction and gradual deformation of the specimens using full-field deformation measurement techniques. The feature tracking results also did not show significant differences between the different R-ratio tests. It can nevertheless be used in the future research to improve our understanding of the deformation distribution in AM porous biomaterials, for example, by comparing porous structures with different types of repeating unit cell. Comparing different lightning conditions or adding non-reflective paint on the specimens could further optimize the feature tracking results, because it was found that the samples were reflective in the presence of a large light source that is needed in combination with the cameras. The increasing deformations of the sample during the test resulted in a change of the reflections that is unfavorable for the feature detection process.

Comparing the S-N curves obtained here with those obtained in a previous study (Amin Yavari et al., 2015) in which similar AM porous biomaterials were tested using a stress ratio of $R=0.1$ shows that the $\mathrm{S}-\mathrm{N}$ curve from that study corresponds with the S-N curve obtained in the current study for $R=0.3$ (and not $R=0.1$ ) (Fig. 5). That might be due to the fact that smaller specimens were used in (Amin Yavari et al., 2013) as compared to those used here $(10 \times 15 \mathrm{~mm}$ instead of $15 \times 20 \mathrm{~mm})$. This can be an indication of a size effect which is known to occur for both smooth and notched specimens (Kloos et al., 1981; Schijve, 2009) as well as for cellular structures (Andrews et al., 2001; Onck et al., 2001). Tests with different sample sizes should be performed to determine whether a size effect is present in the fatigue behavior of this type of AM porous biomaterials.

AM porous biomaterials used for bone grafting or as a part of loadbearing orthopaedic implants are subjected to regular cyclic loading caused by activities such as walking, running, jumping, stair climbing, and various types of sport activities. It is therefore important to design AM porous biomaterials such that they can withstand the experienced fatigue loading. Design for fatigue often requires information regarding the fatigue response of the material under consideration to various loading regimens. For example, it is important to understand the fatigue response of a given material to cyclic loadings with different stress ratios and frequencies. As far as human activities similar to the ones mentioned above are concerned, loading profiles could change considerably from one activity to another (Liikavainio et al., 2007; Mundermann et al., 2008; Taylor et al., 2004; Voloshin, 2000; Voloshin et al., 1998; Zadpoor and Nikooyan, 2006). A wide range of different parameters including, for example, pathological conditions such as osteoarthritis (Liikavainio et al., 2007) and psychosocial stress (Marras et al., 2000) could change musculoskeletal loading. Since it is not possible to determine the $\mathrm{S}-\mathrm{N}$ curves of different materials, empirical relationships such as Goodman and Gerber are used for interpolating between the experimental data and fatigue design of materials under loading conditions not experimentally tested. Since the R-ratio behavior of $\mathrm{AM}$ porous biomaterials does not match those predicted by the empirical relationships used for bulk materials, one may need to develop new empirical relationships for fatigue design of AM porous biomaterials. Further research is needed to determine what the best empirical relationships for fatigue design of AM porous structures are.

\section{Conclusions}

The influence of the R-ratio on the compression-compression fatigue behavior of additively manufactured porous titanium biomaterials based on the diamond unit cell was studied. These porous metal structures are analyzed in this work as if they behave as materials. This means that, stresses, strains and R-ratios are calculated as properties of the cylindrical porous sample, and not of the parent material. Constructing constant life diagrams and comparing the gradual failure under different loading conditions allowed us to study the effects of mean stress and stress amplitude on the constant life diagrams. Feature tracking algorithms were used for full-field deformation measurement during the fatigue tests. Compression-compression S-N curves were found to exhibit the same log-linear trend characteristic of the tension-tension behavior of the bulk material. The S-N curves shifted upwards when the stress ratio increased. The stress amplitude was found to be the dominant factor determining the fatigue lives of the AM porous biomaterials considered here. The constant fatigue life diagrams showed little sensitivity to mean stress, which is very different from what is observed for bulk Ti-6Al-4V and other bulk metals and alloys, meaning that the fatigue behavior of AM porous biomaterials including their R-ratio behavior is not only quantitatively but also qualitatively different from that of bulk materials. One could therefore conclude that relationships similar to the Gerber law that are normally used for the fatigue design of continuous materials are not applicable when designing lattice structures, meaning that new relationships should be developed for that purpose. This result is generalizable to other materials and lattice structures, as it nullifies the assumption that the fatigue design principles used for continuous materials could be directly applied to additively manufactured porous (bio-)materials.

\section{References}

Ahmadi, S.M., et al., 2014. Mechanical behavior of regular open-cell porous biomaterials made of diamond lattice unit cells. J Mech. Behav. Biomed. Mater. 34, 106-115.

Ahmadi, S.M., et al., 2015. Additively manufactured open-cell porous biomaterials made from six different space-filling unit cells: the mechanical and morphological properties. Materials 8 (4), 1871-1896.

Amin Yavari, S., et al., 2013. Fatigue behavior of porous biomaterials manufactured using selective laser melting. Mater. Sci. Eng. 33 (8), 4849-4858.

Amin Yavari, S., et al., 2014. Effects of bio-functionalizing surface treatments on the mechanical behavior of open porous titanium biomaterials. J. Mech. Behav. Biomed. Mater. 36, 109-119.

Amin Yavari, S., et al., 2014. Crystal structure and nanotopographical features on the surface of heat-treated and anodized porous titanium biomaterials produced using selective laser melting. Appl. Surf. Sci. 290, 287-294.

Amin Yavari, S., et al., 2015. Relationship between unit cell type and porosity and the fatigue behavior of selective laser melted meta-biomaterials. $J$ Mech. Behav. Biomed. Mater. 43, 91-100.

Amin Yavari, S., et al., 2016. Antibacterial behavior of additively manufactured porous titanium with nanotubular surfaces releasing silver ions. ACS Appl. Mater. interfaces 8 (27), 17080-17089.

Andrews, E., et al., 2001. Size effects in ductile cellular solids. Part II: experimental results. Int. J. Mech. Sci. 43 (3), 701-713.

B311, A., 2013. Standard Test Method for Density of Powder Metallurgy (PM) Materials Containing Less Than Two Percent Porosity.. ASTM International.

Brenne, F., Niendorf, T., Maier, H., 2013. Additively manufactured cellular structures: impact of microstructure and local strains on the monotonic and cyclic behavior under uniaxial and bending load. J. Mater. Process. Technol. 213 (9), 1558-1564.

Cain, V., et al., 2014. Crack propagation and fracture toughness of Ti6Al4V alloy produced by selective laser melting. Addit. Manuf..

Campoli, G., et al., 2013. Mechanical properties of open-cell metallic biomaterials manufactured using additive manufacturing. Mater. / Des. 49, 957-965.

Cansizoglu, O., et al., 2008. Properties of Ti-6Al-4V non-stochastic lattice structures fabricated via electron beam melting. Mater. Sci. Eng.: A 492 (1), 468-474.

Caron, R.R., et al., 2015. Musculoskeletal stiffness changes linearly in response to increasing load during walking gait. J. Biomech. 48 (6), 1165-1171.

Chahine, G., et al., 2008. The design and production of Ti-6Al-4V ELI customized dental implants. JOM 60 (11), 50-55.

Cheng, X., et al., 2012. Compression deformation behavior of Ti-6Al-4V alloy with cellular structures fabricated by electron beam melting. J. Mech. Behav. Biomed. 
Mater. 16, 153-162.

Gorny, B., et al., 2011. In situ characterization of the deformation and failure behavior of non-stochastic porous structures processed by selective laser melting. Mater. Sci. Eng.: A 528 (27), 7962-7967.

Heinl, P., et al., 2008. Cellular Ti-6Al-4V structures with interconnected macro porosity for bone implants fabricated by selective electron beam melting. Acta Biomater. 4 (5), 1536-1544.

Henry, S.D., et al., 1994. Fatigue Data Book: light Structural Alloys. ASM International.

Hobara, H., et al., 2014. Amputee locomotion: lower extremity loading using running-specific prostheses. Gait posture 39 (1), 386-390.

Hrabe, N.W., et al., 2011. Compression-compression fatigue of selective electron beam melted cellular titanium (Ti-6Al-4V). J. Biomed. Mater. Res. Part B: Appl. Biomater. 99 (2), 313-320.

ISO, ISO 13314: Mechanical Testing of metals - Ductility Testing - Compression Test for Porous and Cellular metals. 2011, ISO

Kloos, K., Buch, A., Zankov, D., 1981. Pure geometrical size effect in fatigue tests with constant stress amplitude and in programme tests. Mater. und Werkst. 12 (2), $40-50$.

Lanning, D.B., Nicholas, T., Haritos, G.K., 2005. On the use of critical distance theories for the prediction of the high cycle fatigue limit stress in notched Ti-6Al-4V. Int. J. fatigue 27 (1), 45-57.

Leuders, S., et al., 2013. On the mechanical behaviour of titanium alloy TiAl6V4 manufactured by selective laser melting: fatigue resistance and crack growth performance. Int. J. Fatigue 48, 300-307.

Li, S., et al., 2012. Compression fatigue behavior of Ti-6Al-4V mesh arrays fabricated by electron beam melting. Acta Mater. 60 (3), 793-802.

Liikavainio, T., et al., 2007. Loading and gait symmetry during level and stair walking in asymptomatic subjects with knee osteoarthritis: importance of quadriceps femoris in reducing impact force during heel strike? Knee 14 (3), 231-238.

Lin, C.Y., et al., 2007. Structural and mechanical evaluations of a topology optimized titanium interbody fusion cage fabricated by selective laser melting process. $J$. Biomed. Mater. Res. Part A 83 (2), 272-279.

Lindemann, J., Wagner, L., 1997. Mean stress sensitivity in fatigue of $\alpha,(\alpha \beta)$ and $\beta$ titanium alloys. Mater. Sci. Eng.: A 234-236, 1118-1121.

Lohman, E.B., Sackiriyas, K.S.B., Swen, R.W., 2011. A comparison of the spatiotemporal parameters, kinematics, and biomechanics between shod, unshod, and minimally supported running as compared to walking. Phys. Ther. Sport 12 (4), 151-163.

Marras, W.S., et al., 2000. The influence of psychosocial stress, gender, and personality on mechanical loading of the lumbar spine. Spine 25 (23), 3045-3054.

Mullen, L., et al., 2009. Selective laser melting: a regular unit cell approach for the manufacture of porous, titanium, bone in-growth constructs, suitable for orthopedic applications. J. Biomed. Mater. Res. Part B: Appl. Biomater. 89 (2), 325-334.

Mundermann, A., et al., 2008. In vivo knee loading characteristics during activities of daily living as measured by an instrumented total knee replacement. J. Orthop. Res. 26 (9), 1167-1172.

Nicholas, T., 2006. High Cycle Fatigue, a mechanics of materials perspective.. Elsevier Science Ltd, Oxford.

Onck, P., Andrews, E., Gibson, L., 2001. Size effects in ductile cellular solids. Part I: modeling. Int. J. Mech. Sci. 43 (3), 681-699.

Ratner, B.D., et al., 2013. Biomaterials science: an introduction to materials in medicine. Academic press.

\section{Rohatgi, A., WebPlotDigitizer. 2015}

Sallica-Leva, E., Jardini, A., Fogagnolo, J., 2013. Microstructure and mechanical behavior of porous Ti-6Al-4V parts obtained by selective laser melting. J. Mech. Behav. Biomed. Mater. 26, 98-108.

Schijve, J., 2009. Fatigue of Structures and Materials. Springer, 621.

van der Stok, J., et al., 2015. Full regeneration of segmental bone defects using porous titanium implants loaded with BMP-2 containing fibrin gels. Eur. Cells Mater. 29, $141-154$.

Taylor, W.R., et al., 2004. Tibio-femoral loading during human gait and stair climbing. J. Orthop. Res. 22 (3), 625-632.

Thijs, L., et al., 2010. A study of the microstructural evolution during selective laser melting of Ti-6Al-4V. Acta Mater. 58 (9), 3303-3312.

Van Hooreweder, B., et al., 2012. Analysis of fracture toughness and crack propagation of Ti6Al4V produced by selective laser melting. Adv. Eng. Mater. 14 (1-2), 92-97.

Van Hooreweder, B., et al., 2016. Improving the fatigue performance of porous metallic biomaterials produced by selective laser melting. Acta Biomater..

Voloshin, A., 2000. The influence of walking speed on dynamic loading on the human musculoskeletal system. Med. Sci. Sport. Exerc. 32 (6), 1156-1159.

Voloshin, A.S., et al., 1998. Dynamic loading on the human musculoskeletal systemeffect of fatigue. Clin. Biomech. 13 (7), 515-520.

Yan, C., et al., 2012. Evaluations of cellular lattice structures manufactured using selective laser melting. Int. J. Mach. Tools Manuf. 62, 32-38.

Zadpoor, A.A., Nikooyan, A.A., 2006. A mechanical model to determine the influence of masses and mass distribution on the impact force during running-a discussion. J. Biomech. 39 (2), 388-390.

Zadpoor, A.A., Sinke, J., Benedictus, R., 2008. Experimental and numerical study of machined aluminum tailor-made blanks. J. Mater. Process. Technol. 200 (1), 288-299.

Zargarian, A., et al., 2016. Numerical simulation of the fatigue behavior of additive manufactured titanium porous lattice structures. Mater. Sci. Eng.: C 60, 339-347. 